\title{
A New Approach to Inflammatory Bowel Disease Therapy
}

\author{
A review of: Steidler L, Hans W, Schotte L, Neirynck S, Obermeier F, Falk W, Fiers W, Remaut E 2000 Treatment of \\ murine colitis by Lactococcus lactis secreting Interleukin-10. Science 289:1352-1355
}

$\mathrm{I}_{\mathrm{n}}^{\mathrm{n}}$ nflammatory bowel disease (IBD) has no definitive cause or known cure. Etiologic theories regarding bacterial involvement have abounded, ranging from a single pathogen, to alterations of the normal flora, to an inappropriate immune response to normal flora. Now, work by Steidler et al. (1) has shown how bacteria can be manipulated and used to treat IBD. Using the bacterium Lactococcus lactis, Steidler et al have genetically engineered these bacteria to secrete the anti-inflammatory cytokine, interleukin-10 (IL-10). In these studies, the authors showed that daily intragastric inoculation of L. lactis-mIL-10 for 14 days resulted in attenuated mucosal inflammation in mice with dextransulfate induced colitis and prevented the development of adenocarcinoma. Using a genetically altered rodent model of colitis, the authors also demonstrated that treatment of 3-wk-old IL-10 gene-deficient mice with daily intragastric inoculation of L. lactismIL-10 for 4 weeks completely prevented colitis.

The use of L. lactis as a vector to secrete biologically active molecules is well thought-out. L. lactis is a Grampositive bacterium, which is classified as "safe" following its long history of use in fermented milk products. L. lactis is a nonpathogenic bacterium which does not multiply nor colonize in humans in vivo. However, the demonstra-

\section{Karen Madsen}

tion that these bacteria are able to deliver cytokines directly to the mucosa and that this strategy is therapeutically effective in two very different mouse models of colitis, opens the door for a myriad of new therapeutic strategies.

In patients with IBD, cytokines produced by subsets of $\mathrm{T}$ cells appear to critically influence immune responses and provide a target for immunotherapy treatments. Whereas Crohn's disease is associated with a predominance of TH1 cytokines (i.e. TNF- $\alpha$, IFN- $\gamma$, IL-12), ulcerative colitis tends to be associated with $\mathrm{TH} 2$ cytokines (i.e. IL-4, IL-5). Current biological based therapies for Crohn's disease are aimed at reducing the levels of $\mathrm{TH} 1$ cytokines with either antibodies to TNF- $\alpha$ (2) or recombinant IL-10. Thus, the decision by Steidler et al. (1) to use IL-10 as treatment for IBD is an exciting new concept. Administration of IL-10 has proven therapeutic benefit in several mouse models of IBD and in patients with Crohn's disease. However, the clinical usefulness of current immunotherapy is limited because of the exceptionally high cost, a lack of organ specificity, and undesirable side effects. The use of genetically engineered bacteria to deliver cytokines such as IL-10 directly to the gut would be convenient and cost-effective, and may well avoid the problems associ- ated with systemic exposure to cytokines; all of which would have a definite appeal to patients, their families and health-care providers.

While this study opens up a whole new approach to the treatment of inflammatory bowel diseases, safety of these bacteria for use in humans must be assured and will need to be addressed. Could these bacteria be spread person-to-person? What would be the effect of such transmission? Could these genetically engineered bacteria pass their IL-10 synthesizing ability to a pathogenic or a colonizing species? If so, what would be the outcome? Are these bacteria safe for use in immunocompromised individuals or neonates? These and many more questions await answers. Until then, what we have is an exciting new approach to the treatment of IBD using a "cost-effective localized delivery system of a therapeutic agent that is actively synthesized in situ by food-grade bacteria."

\footnotetext{
1. Steidler L, Hans W, Schotte L, Neirynck S, Obermeier F, Falk W, Fiers W, Remaut E 2000 Treatment of murine colitis by Lactococcus lactis secreting Interleukin-10. Science 289:1352-1355

2. Bell SJ, Kamm MA 2000 The clinical role of antiTNFalpha antibody treatment in Crohn's disease. Alimen Pharmacol Ther 14(5):501-514
}

University of Alberta Faculty of Medicine and Dentistry

Division of Gastroenterology

536 Newton Building

Edmonton, Alberta, Canada

T6G $2 C 2$ 